\title{
Periodic Input Response of a Second-Order Digital Filter With Two's Complement Arithmetic
}

\author{
Xiangjun Li, Xinghuo Yu, Fellow, IEEE, Changhong Wang, and Bingo Wing-Kuen Ling, Senior Member, IEEE
}

\begin{abstract}
The dynamic behaviors of a nonlinear second-order digital filter with two's complement arithmetic under periodic inputs are explored. The conditions for avoiding overflow are derived. Various dynamic periodic responses are analyzed, accompanied by numerous simulation examples.
\end{abstract}

Index Terms-Chaos, dynamic symbolic sequence, nonlinear digital filter, overflow, switching system.

\section{INTRODUCTION}

C HAOS can happen in a marginally stable second-order digital filter with two's complement arithmetic, and various chaotic behaviors were observed [1]-[4]. Since then, there has been substantial further research in this particular topic. In [5], an overall analysis of complex dynamics in the second-order digital filter with overflow nonlinearity was given. In [6], periodic behaviors were completely studied with the analysis of the admissibility of periodic symbolic sequences. An attempt was made to find the exact classification of the domain of initial states according to different symbolic sequences. Aperiodic behaviors were observed through simulation examples. The aforementioned results are concerned with the autonomous system case. In [7] and [8], the dynamic behaviors of the second-order filter under the step and sinusoidal inputs were considered. Methods of the affine transformation and frequency-domain technique were employed in explaining the trajectory behaviors. In addition to the analysis of periodic and aperiodic dynamic behaviors, conditions for avoiding overflow were given.

This brief further explores the dynamic behaviors of the second-order digital filter with two's complement arithmetic under general periodic inputs, which have not been done before. Conditions for avoiding overflow are first derived. Various periodic behaviors are then analyzed, accompanied by numerous simulation results.

This brief is organized as follows. Section II is the problem description. In Section III, the conditions for avoiding overflow are derived. Section IV explores the various behaviors when overflow occurs, such as the admissibility of periodic symbolic sequence and the relationship between system parameters and periodicity. Conclusion is drawn in Section V.

Manuscript received April 26, 2008; revised July 30, 2008. Current version published March 20, 2009. This paper was recommended by Associate Editor M. Chakraborty.

$\mathrm{X}$. Li and C. Wang are with the Department of Control Science and Engineering, Harbin Institute of Technology, Harbin 150001, China (e-mail: xiangjun.li@rmit.edu.au; cwang@hit.edu.au).

$\mathrm{X}$. Yu is with the School of Electrical and Computer Engineering, Royal Melbourne Institute of Technology (RMIT University), Melbourne, Vic. 3001, Australia (e-mail: x.yu@rmit.edu.au).

B. W.-K. Ling is with the Department of Electronic Engineering, King's College London, London WC2R 2LS, U.K. (e-mail: wing-kuen.ling@kcl.ac.uk). Digital Object Identifier 10.1109/TCSII.2009.2015351

\section{PRoblem Description}

Digital filters have finite binary operations and registers. When a calculation produces a result that is greater in magnitude than the registers can store, overflow happens. With two's complement arithmetic, the digital filters handle overflow by counting backward and wrapping around the overflowed number. The marginally stable second-order digital filter with two's complement arithmetic can be described as [8]

$$
\mathbf{x}(k+1)=\mathbf{A} \cdot \mathbf{x}(k)+\mathbf{B} \cdot u(k)+\mathbf{B}_{s} \cdot s_{k}
$$

where

$$
\mathbf{A}=\left[\begin{array}{cc}
0 & 1 \\
-1 & a
\end{array}\right] \quad \mathbf{B}=\left[\begin{array}{l}
0 \\
1
\end{array}\right] \quad \mathbf{B}_{s}=\left[\begin{array}{l}
0 \\
2
\end{array}\right]
$$

with $a=2 \cos (\Omega), 0<\Omega<\pi$, and $s_{k}$ is an integer such that

$$
\mathbf{x}(k) \in \mathbf{I}^{2} \triangleq\left\{\left[\begin{array}{l}
x_{1}(k) \\
x_{2}(k)
\end{array}\right]:-1 \leq x_{1}<1,-1 \leq x_{2}<1\right\} .
$$

In this brief, the coefficient quantization and round-off noise are supposed to be null.

More general than the cases in [7] and [8], the input $u(k)$ is a periodic signal with period $m$, which can be expressed as the Fourier series

$$
u(k)=\sum_{i=0}^{m-1}\left(a_{i} \sin (i \omega k)+b_{i} \cos (i \omega k)\right)(k \geq 0)
$$

where $a_{i}$ and $b_{i}$ are the Fourier coefficients, and $\omega=2 \pi / m$. We assume that $\omega \neq k \pi$; otherwise, the system will be reduced to a step-response case, and the relevant results can be found in [7].

This nonlinear digital filter can be viewed as a nonlinear map from $\mathbf{I}^{2}$ to the space composed of dynamic symbolic sequences denoted as $\mathbf{s}=\left\{s_{0}, s_{1}, \ldots\right\}$. The mapping function is denoted as $\mathcal{S}$. A symbolic sequence $\mathbf{s}$ is admissible if there exists a point $\mathbf{x} \in \mathbf{I}^{2}$ such that $\mathcal{S}(\mathbf{x})=\mathbf{s}$.

The following notations will be used throughout this brief:

$$
\begin{aligned}
\mathbf{T}_{\Omega} & =\left[\begin{array}{cc}
1 & 0 \\
\cos (\Omega) & \sin (\Omega)
\end{array}\right] \\
\mathbf{T}_{i, \omega} & =\left[\begin{array}{cc}
1 & 0 \\
\cos (i \omega) & \sin (i \omega)
\end{array}\right] \\
\hat{\mathbf{A}}_{\Omega} & =\left[\begin{array}{cc}
\cos (\Omega) & \sin (\Omega) \\
-\sin (\Omega) & \cos (\Omega)
\end{array}\right] \\
\hat{\mathbf{A}}_{i, \omega} & =\left[\begin{array}{cc}
\cos (i \omega) & \sin (i \omega) \\
-\sin (i \omega) & \cos (i \omega)
\end{array}\right]
\end{aligned}
$$




$$
\begin{aligned}
\mathbf{x}_{0}^{*} & =b_{0} /(2-a)\left[\begin{array}{l}
1 \\
1
\end{array}\right] \\
\mathbf{x}_{1}^{*} & =b_{\frac{m}{2}} /(2+2 \cos \Omega)\left[\begin{array}{c}
-1 \\
1
\end{array}\right](m \text { is even }) \\
\mathbf{x}_{i, 1}^{*} & =\left(a_{i} \sin (i \omega)\right) /(2 \cos (i \omega)-2 \cos (\Omega))\left[\begin{array}{l}
1 \\
0
\end{array}\right] \\
\mathbf{x}_{i, 2}^{*} & =b_{i} /(2 \cos (i \omega)-2 \cos (\Omega))\left[\begin{array}{c}
\cos (i \omega) \\
1
\end{array}\right], \\
\mathbf{\Psi}_{k} & =\left(\mathbf{A}^{k-1} \mathbf{B}, \mathbf{A}^{k-2} \mathbf{B}, \ldots, \mathbf{A B}, \mathbf{B}\right) \\
\mathbf{\Phi}_{k} & =\left(\mathbf{A}^{k-1} \mathbf{B}_{s}, \mathbf{A}^{k-2} \mathbf{B}_{s}, \ldots, \mathbf{A B} \mathbf{B}_{s}, \mathbf{B}_{s}\right)
\end{aligned}
$$

$\|\cdot\|_{\infty}$ is the infinity norm, and $\|\cdot\|$ represents the 2-norm. Given $\mathbf{s}=\left\{s_{0}, s_{1}, s_{2} \ldots\right\}$ and $\mathbf{u}=\{u(0), u(1), u(2), \ldots\}$, define $\mathbf{s}_{u}=\left\{2 s_{0}+u(0), 2 s_{1}+u(1), \ldots\right\}$.

\section{NONOVERfLOW CASE}

In engineering applications, filters are assumed to be functional within the linear areas, and overflow should completely be avoided. It is meaningful to find conditions under which there will be no overflow. We will derive these conditions in this section.

First, system (1) can be rewritten into the following convenient forms [ $m$ is defined in (4)] [9]:

- If $m$ is odd

$$
\begin{gathered}
\mathbf{x}(k)=\mathbf{T}_{\Omega} \hat{\mathbf{A}}_{\Omega}^{k} \mathbf{T}_{\Omega}^{-1}\left(\mathbf{x}(0)-\mathbf{x}_{0}^{*}+\sum_{i=1}^{m-1}\left(\mathbf{x}_{i, 1}^{*}-\mathbf{x}_{i, 2}^{*}\right)\right) \\
+\sum_{i=1}^{m-1} \mathbf{T}_{i, \omega} \hat{\mathbf{A}}_{i, \omega}^{k} \mathbf{T}_{i, \omega}^{-1}\left(\mathbf{x}_{i, 2}^{*}-\mathbf{x}_{i, 1}^{*}\right)+\mathbf{\Psi}_{k} \mathbf{s}^{T}+\mathbf{x}_{0}^{*} .
\end{gathered}
$$

- If $m$ is even

$$
\begin{aligned}
\mathbf{x}(k)= & \mathbf{T}_{\Omega} \hat{\mathbf{A}}_{\Omega}^{k} \mathbf{T}_{\Omega}^{-1}\left(\mathbf{x}(0)-\mathbf{x}_{0}^{*}+\mathbf{x}_{1}^{*}+\sum_{i=1, i \neq \frac{m}{2}}^{m-1}\left(\mathbf{x}_{i, 1}^{*}-\mathbf{x}_{i, 2}^{*}\right)\right) \\
& +\sum_{i=1, i \neq \frac{m}{2}}^{m-1} \mathbf{T}_{i, \omega} \hat{\mathbf{A}}_{i, \omega}^{k} \mathbf{T}_{i, \omega}^{-1}\left(\mathbf{x}_{i, 2}^{*}-\mathbf{x}_{i, 1}^{*}\right) \\
& +\mathbf{\Psi}_{k} \mathbf{s}^{T}+\mathbf{x}_{0}^{*}+(-1)^{k-1} \mathbf{x}_{1}^{*} .
\end{aligned}
$$

The reason why there are two forms according to different $m$ is that when $m$ is even and $i=m / 2, \mathbf{T}_{i, \omega}$ is not invertible. Since $\hat{\mathbf{A}}_{\Omega}^{k}$ and $\hat{\mathbf{A}}_{\omega}^{i}$ are rotation matrices, from (5) and (6), we can find the number of oscillatory components in the state trajectories. In the following, we develop sufficient conditions on initial states such that overflow will not happen.

Theorem 1: If the initial states satisfy either of the following conditions:

a) If $m$ is odd

$$
\begin{aligned}
\| \mathbf{T}_{\Omega}^{-1} & \left(\mathbf{x}(0)-\mathbf{x}_{0}^{*}+\sum_{i=1}^{m-1}\left(\mathbf{x}_{i, 1}^{*}-\mathbf{x}_{i, 2}^{*}\right)\right) \| \\
& <1-\sum_{i=1}^{m-1}\left\|\mathbf{T}_{i, \omega}^{-1}\left(\mathbf{x}_{i, 2}^{*}-\mathbf{x}_{i, 1}^{*}\right)\right\|-\left\|\mathbf{x}_{0}^{*}\right\|_{\infty} .
\end{aligned}
$$

b) If $m$ is even

$$
\begin{aligned}
\| & \mathbf{T}_{\Omega}^{-1}\left(\mathbf{x}(0)-\mathbf{x}_{0}^{*}+\mathbf{x}_{1}^{*}+\sum_{i=1, i \neq \frac{m}{2}}^{m-1}\left(\mathbf{x}_{i, 1}^{*}-\mathbf{x}_{i, 2}^{*}\right)\right) \| \\
& <1-\sum_{i=1}^{m-1}\left\|\mathbf{T}_{i, \omega}^{-1}\left(\mathbf{x}_{i, 2}^{*}-\mathbf{x}_{i, 1}\right)^{*}\right\| \\
& -\max \left\{\left\|\mathbf{x}_{0}^{*}-\mathbf{x}_{1}^{*}\right\|_{\infty},\left\|\mathbf{x}_{0}^{*}+\mathbf{x}_{1}^{*}\right\|_{\infty}\right\} .
\end{aligned}
$$

Then, overflow will not happen.

Proof: We only prove condition a) because the proof of condition b) is the same. Since $\mathbf{x}(k) \in \mathbf{I}^{2}$, then $s(k)=0$. Its equivalent form is

$$
\begin{aligned}
\|\mathbf{x}(k)\|_{\infty} & =\| \mathbf{T}_{\Omega} \mathbf{A}_{\Omega}^{k} \mathbf{T}_{\Omega}^{-1}\left[\mathbf{x}(0)-\mathbf{x}_{0}^{*}+\sum_{i=1}^{m-1}\left(\mathbf{x}_{i, 1}^{*}-\mathbf{x}_{i, 2}^{*}\right)\right] \\
& +\sum_{i=1}^{m-1} \mathbf{T}_{i, \omega} \mathbf{A}_{i, \omega}^{k} \mathbf{T}_{i, \omega}^{-1}\left(\mathbf{x}_{i, 2}^{*}-\mathbf{x}_{i, 1}^{*}\right)+\mathbf{x}_{0}^{*} \|_{\infty}<1 .
\end{aligned}
$$

$\mathbf{x}(k)$ is the composition of several oscillatory components. On the phase portrait, it is the summation of several rotating telescopic vectors. Let us take item $\mathbf{T}_{\Omega} \hat{\mathbf{A}}_{\Omega}^{k} \mathbf{T}_{\Omega}^{-1}[\mathbf{x}(0)-$ $\left.\mathbf{x}_{0}^{*}+\sum_{i=1}^{m-1}\left(\mathbf{x}_{i, 1}^{*}-\mathbf{x}_{i, 2}^{*}\right)\right]$ as an example. The image of vector $\mathbf{T}_{\Omega}^{-1}\left[\mathbf{x}(0)-\mathbf{x}_{0}^{*}+\sum_{i=1}^{m-1}\left(\mathbf{x}_{i, 1}^{*}-\mathbf{x}_{i, 2}^{*}\right)\right]$ under rotational map $\hat{\mathbf{A}}_{\Omega}^{k}$ is a set of points located on a circle whose radius is $\rho=$ $\left\|\mathbf{T}_{\Omega}^{-1}\left[\mathbf{x}(0)-\mathbf{x}_{0}^{*}+\sum_{i=1}^{m-1}\left(\mathbf{x}_{i, 1}^{*}-\mathbf{x}_{i, 2}^{*}\right)\right]\right\|$. This circle is transformed into an ellipse with a $45^{\circ}$ inclination by $\mathbf{T}_{\Omega}$. Furthermore, this ellipse is restricted in a square box whose sides have a length of $2 \rho$ [1]. Thus, we can strengthen condition (9) as

$$
\begin{aligned}
\|\mathbf{x}(k)\|_{\infty} \leq & \left\|\mathbf{T}_{\Omega}^{-1}\left[\mathbf{x}(0)-\mathbf{x}_{0}^{*}+\sum_{i=1}^{m-1}\left(\mathbf{x}_{i, 1}^{*}-\mathbf{x}_{i, 2}^{*}\right)\right]\right\| \\
& +\sum_{i=1}^{m-1}\left\|\mathbf{T}_{i, \omega}^{-1}\left(\mathbf{x}_{i, 2}^{*}-\mathbf{x}_{i, 1}^{*}\right)\right\|+\left\|\mathbf{x}_{0}^{*}\right\|_{\infty}<1
\end{aligned}
$$

or as

$$
\begin{aligned}
\| \mathbf{T}_{\Omega}^{-1}(x(0) & \left.-\mathbf{x}_{0}^{*}+\sum_{i=1}^{m-1}\left(\mathbf{x}_{i, 1}^{*}-\mathbf{x}_{i, 2}^{*}\right)\right) \| \\
& <1-\sum_{i=1}^{m-1}\left\|\mathbf{T}_{i, \omega}^{-1}\left(\mathbf{x}_{i, 2}^{*}-\mathbf{x}_{i, 1}^{*}\right)\right\|-\left\|\mathbf{x}_{0}^{*}\right\|_{\infty} .
\end{aligned}
$$

Remark 1: The region described by condition (11) is an ellipse on the phase plane whose center is located at $\mathbf{x}_{0}^{*}-$ $\sum_{i=1}^{m-1}\left(\mathbf{x}_{i, 2}^{*}-\mathbf{x}_{i, 1}^{*}\right)$. Since the condition is only sufficient, this area is only a subset of the nonoverflow area.

Remark 2: Conditions (7) and (8) are a generalization of the corresponding results in [8], which tells us how the inputs affect the nonoverflow region. For example, in the definition of $\mathbf{x}_{i, 1}^{*}$ and $\mathbf{x}_{i, 2}^{*}, 2 \cos (i \omega)-2 \cos (\Omega)$ appears in the denominator. It means that if $\Omega$ is close to one of the discrete frequency components in the input, overflow will easily happen. This is because the region abruptly shrinks or even disappears. All in all, the estimated nonoverflow area will shrink due to the 
TABLE I

Nonoverflow AREA Estimation Results

\begin{tabular}{l|c|c|c|c|c|c}
\hline & \multicolumn{3}{|c|}{$\Omega=2 \pi / 5$} & \multicolumn{3}{c}{$\Omega=1.2$} \\
\hline & center & semimajor axis & semiminor axis & center & semimajor axis & semiminor axis \\
\hline autonomous case & $(0.0,0.0)$ & 1.1441 & 0.8313 & $(0.0,0.0)$ & 1.1672 & 0.7985 \\
\hline periodic input case & $(0.0736,0.0878)$ & 0.9715 & 0.7058 & $(0.0820,0.0953)$ & 0.9806 & 0.6708 \\
\hline
\end{tabular}

Partition Graph $(\Omega=2 \pi / 5)$

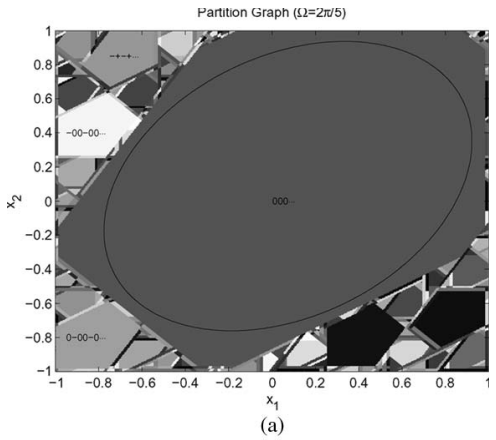

Partition Graph $(\Omega=1.2$

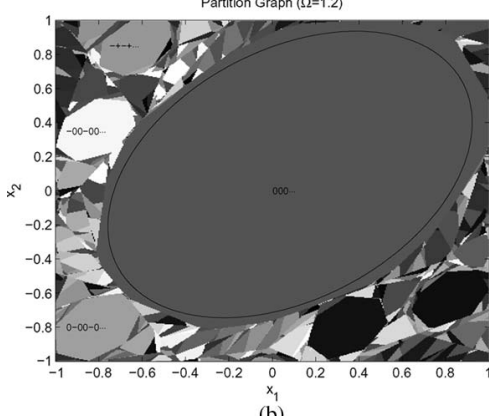

Fig. 1. Partition according to different symbolic sequences and the estimated nonoverflow area. (a) $\Omega=2 \pi / 5$. Periodic input case. Partitioned according to the first 30 symbols of admissible sequences. (b) $\Omega=1.2$. Periodic input case. Partitioned according to the first 30 symbols of admissible sequences.

periodic input, as compared with the autonomous case. As shown in inequalities (7) and (8), the "diameter" of the nonoverflow area is decreased by the items on the right side of the inequalities, which are dependent on the periodic input.

To verify the preceding remarks, an example is presented below.

Example 1: Set $\Omega=2 \pi / 5$ and $\Omega=1.2$, respectively. Let other parameters be $b_{0}=0.123, a_{1}=-0.01, b_{1}=0.014, a_{2}=$ $0.02, b_{2}=-0.012$, and $\omega=2 \pi / 3$. Thus, the input is period- 3 . Results are listed in Table I and are depicted in Fig. 1. It can be seen that the areas tagged with "000 ..." in Fig. 1(a) and (b) are the nonoverflow areas, and the ellipses located in them are the areas derived from applying condition (7). Other areas with different gray scales are the areas corresponding to different symbolic sequences. Thus, $\mathbf{I}^{2}$ is partitioned according to different admissible symbolic sequences. Some observations are made.

1) Admissible symbolic sequences in the autonomous case are not necessarily admissible in the periodic-input case.

2) The area corresponding to a certain symbolic sequence may be shifted, amplified, or shrunk because of the periodic inputs.

3) The partition graph for the autonomous case is symmetric (even with fractal), which was proved in [5]. However, when a periodic input is present, the map (1) is no longer symmetric, which leads to an asymmetric partition.

In the following, we will explore the overflow case.

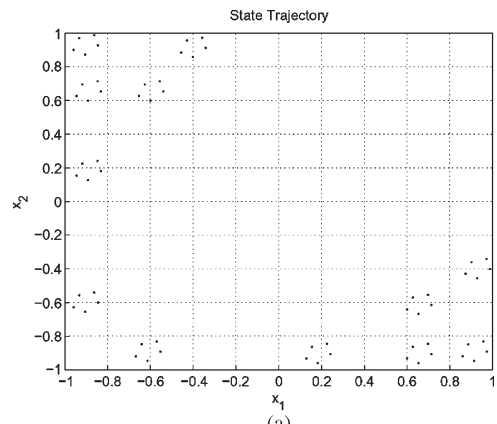

$x_{1}$

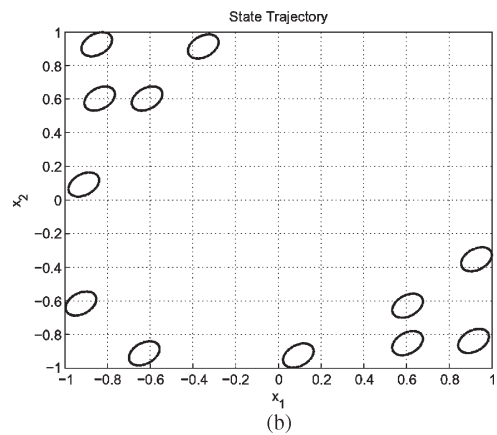

Fig. 2. (a) State trajectory with initial state $\mathbf{x}(0)=\left[\begin{array}{ll}-0.6 & 0.6\end{array}\right]^{T}, \omega=$ $2 \pi / 3$, and $\Omega=2 \pi / 5$. Symbolic sequence $\{-+-+-+-00-00 \cdots\}$. (b) State trajectory with initial state $\mathbf{x}(0)=\left[\begin{array}{ll}-0.65 & 0.65\end{array}\right]^{T}, \omega=2 \pi / 3$, and $\Omega=1.2$. Symbolic sequence $\{-+-+-+-00-00 \cdots\}$.

\section{OVERFLOW CASE}

When overflow occurs, there are two typical dynamic behaviors, as shown in Fig. 2(a) and (b), where the parameters are the same as Example 1. In Fig. 2(a) (with $\Omega=2 \pi / 5$ and $\left.\mathbf{x}(0)=\left[\begin{array}{ll}-0.6 & 0.6\end{array}\right]^{T}\right)$, the points are grouped into 12 clusters, each composed of five points. The period of the symbolic sequence is 12 , i.e., $\{-+-+-+-00-00 \cdots\}$. Fig. 2(b) (with $\Omega=1.2$ and $\mathbf{x}(0)=\left[\begin{array}{ll}-0.65 & 0.65\end{array}\right]^{T}$ ) exhibits 12 continuous ellipses scattered on the phase plane. The period of the symbolic sequence is 12 , i.e., $\{-+-+-+-00-00 \cdots\}$.

There are two questions that need to be answered.

1) What symbolic sequences can be generated? Or equivalently, what symbolic sequences are admissible?

2) How many clusters will be there, and where are they located?

In [1], a necessary and sufficient condition on the admissibility of a given periodic sequence was given for the autonomous case. However, when the input was considered, only necessary conditions were derived in [9]. In this section, inspired by the existing results, we propose a necessary and sufficient condition for the admissibility when periodic input signals are introduced.

Before we present the main result, a lemma is first introduced, which will later be used.

Lemma 1: Given a symbolic sequence $\mathbf{s}=\left\{s_{0}, s_{1}, \ldots\right\}$, define the set

$$
\Omega_{s}=\{\mathbf{x} \mid \mathcal{S}(\mathbf{x})=\mathbf{s}\} .
$$

Therefore, this set is convex. 
Proof: Suppose that $\mathbf{x}_{1}$ and $\mathbf{x}_{2}$ are two arbitrary points in $\Omega_{s}$. The necessary and sufficient condition of $\mathbf{x}(0) \in \Omega_{s}$ is

$$
\begin{aligned}
\mathbf{x}(k)= & \mathbf{A}^{k} \mathbf{x}(0)+\boldsymbol{\Phi}_{k}\left[\begin{array}{lll}
s_{0} s_{1} & \cdots & s_{k-1}
\end{array}\right]^{T} \\
& +\mathbf{\Psi}_{k}\left[\begin{array}{lll}
u(0) u(1) & \cdots & u(k-1)
\end{array}\right]^{T} \\
& \in \mathbf{I}_{2} \quad(\forall k \geq 0) .
\end{aligned}
$$

Thus, to prove that $\Omega_{s}$ is a convex set, we just have to prove that for every $0 \leq \lambda \leq 1$, point $\lambda \mathbf{x}_{1}+(1-\lambda) \mathbf{x}_{2}$ satisfies the preceding condition.

Since both $\mathbf{x}_{1}$ and $\mathbf{x}_{2}$ are in $\Omega_{s}$, then they should satisfy condition (13). That is

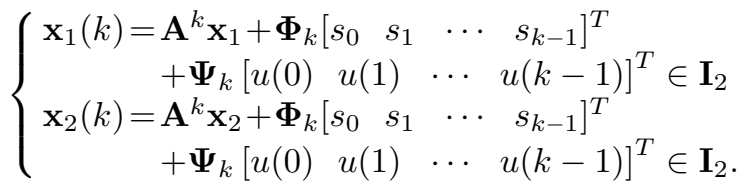

We notice that $\mathbf{I}_{2}$ is a convex set itself; thus, for $0 \leq \lambda \leq 1$, we have

$$
\lambda \mathbf{x}_{1}(k)+(1-\lambda) \mathbf{x}_{2}(k) \in \mathbf{I}_{2} \quad(\forall k \geq 0)
$$

which is

$$
\begin{aligned}
& \mathbf{A}^{k}\left(\lambda \mathbf{x}_{1}+(1-\lambda) \mathbf{x}_{2}\right)+\mathbf{\Phi}_{k}\left[\begin{array}{llll}
s_{0} & s_{1} & \cdots & s_{k-1}
\end{array}\right]^{T} \\
& +\boldsymbol{\Psi}_{k}\left[\begin{array}{lllll}
u(0) & u(1) & \cdots & u(k-1)
\end{array}\right]^{T} \in \mathbf{I}_{2}
\end{aligned}
$$

Following condition (13), the symbolic sequence corresponding to point $\mathbf{z}=\lambda \mathbf{x}_{1}+(1-\lambda) \mathbf{x}_{2}$ is $\mathbf{s}$, which is the same as $\mathbf{x}_{1}$ and $\mathbf{x}_{2}$. Hence, $\Omega_{s}$ is a convex set.

Theorem 2: For system (1), given a period- $L_{u}$ input signal $u(k)$ and a period- $L_{s}$ symbolic sequence

$$
\mathbf{s}=\left\{s_{0}, s_{1}, \ldots, s_{L_{s}-1}, s_{0}, s_{1}, \ldots, s_{L_{s}-1}, \ldots\right\} .
$$

Let $L=\operatorname{lcm}\left(L_{u}, L_{s}\right)$ (lcm stands for the least common multiple). If $\mathbf{I}-\mathbf{A}^{L}$ is invertible, then $\mathbf{s}$ is admissible if and only if $\mathbf{z}_{i} \in \mathbf{I}_{2}\{i=0,1, \ldots, L-1\}$, where $\mathbf{z}_{i}$ are defined as follows:

$$
\mathbf{z}_{i}=\left(\mathbf{I}-\mathbf{A}^{L}\right)^{-1} \mathbf{\Psi}_{L} \mathbf{s}_{u}^{i}
$$

with

$\mathbf{s}_{u}^{i}=\left\{\mathbf{s}_{u}(i), \mathbf{s}_{u}(i+1), \ldots, \mathbf{s}_{u}(L-1), \mathbf{s}_{u}(0), \mathbf{s}_{u}(1), \ldots, \mathbf{s}_{u}(i-1)\right\}$.

Proof: Sufficiency: Expanding (17) yields

$$
\begin{aligned}
& \mathbf{z}_{1}=\mathbf{A} \mathbf{z}_{0}+\mathbf{B} u(0)+\mathbf{B}_{s} s_{0} \\
& \mathbf{z}_{2}=\mathbf{A} \mathbf{z}_{1}+\mathbf{B} u(1)+\mathbf{B}_{s} s_{1} \\
& \vdots \\
& \mathbf{z}_{0}=\mathbf{A} \mathbf{z}_{(L-1)}+\mathbf{B} u(L-1)+\mathbf{B}_{s} s_{L-1} .
\end{aligned}
$$

Hence, $\mathbf{z}(k+m L)=\mathbf{z}(k)$. If $\mathbf{z}_{i} \in \mathbf{I}_{2}$, then $\mathbf{s}(i)=s_{i}$. Setting the initial state as $\mathbf{z}_{0}$, we can get the desired sequence.

Necessity: If the given symbolic sequence is admissible, then there will be at least one point $\mathbf{x}(0)$ such that $\mathcal{S}(\mathbf{x}(0))=\mathbf{s}$. Since $\left(\mathbf{I}-\mathbf{A}^{L}\right)$ is invertible, then

$$
\begin{aligned}
\mathbf{x}(k+m L)= & \mathbf{A}^{m L} \mathbf{x}(k)+\sum_{i=0}^{m L-1} \mathbf{A}^{m L-1-i} \mathbf{B} \mathbf{s}_{u}(k+i) \\
= & \mathbf{A}^{m L} \mathbf{x}(k)+\left(\mathbf{A}^{L}-\mathbf{I}\right)^{-1}\left(\mathbf{A}^{m L}-\mathbf{I}\right) \\
& \times \sum_{i=0}^{L-1} \mathbf{A}^{L-1-i} \mathbf{B} \mathbf{s}_{u}(k+i) .
\end{aligned}
$$

TABLE II

Admissible Symbolic SEQUences With A PERIOD LESS THAN $10(\Omega=2 \pi / 5)$

\begin{tabular}{l|c}
\hline Admissible Sequences & Initial state $\mathbf{Z}_{0}$ \\
\hline 0 & $(0.087767568145748,0.105678664151786)$ \\
\hline+- & $(0.851699590645959,-0.658253358348424)$ \\
\hline-+ & $(-0.676164454354463,0.869610686651996)$ \\
\hline-00 & $(-0.806659622854168,0.447319450651660)$ \\
\hline $0-0$ & $(-0.806659622854168,-0.788748526848130)$ \\
\hline $00-$ & $(0.429408354645622,-0.788748526848130)$ \\
\hline+00 & $(0.982194759145663,-0.235962122348088)$ \\
\hline$-00-00-+$ & $(-0.937154791353874,0.788960237151534)$ \\
\hline$-00-+-00$ & $(-0.937154791353874,0.025028214651324)$ \\
\hline$-+-00-00$ & $(-0.465018836354295,0.788960237151534)$ \\
\hline $0-00-+-0$ & $(-0.465018836354294,-0.919243695347835)$ \\
\hline $0-+-00-0$ & $(-0.937154791353874,-0.447107740348256)$ \\
\hline $00-00-+-$ & $(0.771049141145496,-0.447107740348256)$ \\
\hline $00-+-00-$ & $(0.007117118645285,-0.919243695347835)$ \\
\hline$+-00-00-$ & $(0.771049141145496,-0.919243695347835)$ \\
\hline &
\end{tabular}

Subtracting $\mathbf{z}_{k}$ from both sides of (19) yields

$$
\mathbf{x}(k+m L)-\mathbf{z}_{k}=\mathbf{A}^{m L}\left(\mathbf{x}(k)-\mathbf{z}_{k}\right) .
$$

This equation tells us that points $\mathbf{x}(k+m L)$ are in fact located on the same ellipse centered at $\mathbf{z}_{k}$. On the other hand, $\Omega_{s}$ is a convex set (Lemma 1). Hence, $\mathbf{z}_{0} \in \Omega_{s}$, which means that $\mathcal{S}\left(\mathbf{z}_{0}\right)=\mathbf{s}$. This completes the necessity proof.

Example 2: Let $\Omega=2 \pi / 5, b_{0}=0.123, a_{1}=-0.01, b_{1}=$ $0.014, a_{2}=0.02, b_{2}=-0.012$, and $\omega=2 \pi / 3$; thus, the input is period-3. Using the aforementioned admissibility theorem, we find all admissible periodic symbolic sequences whose periods are less than or equal to 8 , as well as their corresponding initial states. They are listed in Table II.

Theorem 2 provides a mechanism for constructing algorithms to find admissible symbolic sequences.

With this newly defined $\mathbf{s}_{u}$, results in [1] and [6] can easily be translated into our framework by replacing $\mathbf{s}$ with $\mathbf{s}_{u}$. In the following, such results about periodicity are summarized into a theorem using our framework.

Theorem 3: Assume $\Omega=2 r \pi$.

1) If $r=q / p(\operatorname{lcm}(p, q)=1$, where $p$ and $q$ are positive integers) is rational. Let us assume that $\mathbf{s}_{u}$ corresponding to the trajectory starting at $\mathbf{x}(0)$ has period $L$. Define $n$ as the least common multiple of $p$ and $L$, i.e., $n=$ $\operatorname{lcm}(p, L)$, and periodically extend $\mathbf{s}_{u}$ so that the sequence $\mathbf{s}_{u}$ has length $n$. Then

a)

$$
y_{s}=\boldsymbol{\Psi}_{n}\left[\mathbf{s}_{u}(0) \quad \mathbf{s}_{u}(1) \quad \cdots \quad \mathbf{s}_{u}(n-1)\right]^{T}=0 .
$$

$\mathbf{x}(0)$ is periodic with period $n$ (not necessary minimum).

b) If $L$ is not a multiple of $p$. Then

i) If $\mathbf{x}(0) \neq \mathbf{z}_{0}$, then the trajectory starting at $\mathbf{x}(0)$ has the minimum period $n=\operatorname{lcm}(p, L)$. Furthermore, the points of the periodic trajectory are grouped into $L$ elliptic sets centered at $\mathbf{z}_{i}$, and each of the elliptic sets is composed of $n / L$ points. The $L$ ellipses are congruent to each other with a semimajor axis $(1+\cos (\Omega))^{1 / 2} \rho$ and a semiminor axis $(1-\cos (\Omega))^{1 / 2} \rho$, where $\rho=\left\|\mathbf{T}^{-1}\left(\mathbf{x}(0)-\mathbf{z}_{0}\right)\right\|$.

ii) If $\mathbf{x}(0)=\mathbf{z}_{0}$, then the trajectory starting at $\mathbf{x}(0)$ has the minimum period $L$ and $\mathbf{x}(i)=\mathbf{z}_{i}(\bmod L)$.

c) If $L$ is a multiple of $p$, then $L$ is the minimum period of $\mathbf{x}(0)$. 
2) If $r$ is irrational, then the trajectory is dense on $L$ ellipses centered at $\mathbf{z}_{i}$.

Proof:

1a) Since

$$
\mathbf{A}^{n}=\left(\mathbf{T} \hat{\mathbf{A}}_{\Omega} \mathbf{T}^{-1}\right)^{n}=\mathbf{T} \hat{\mathbf{A}}_{\Omega}^{n} \mathbf{T}^{-1}=\mathbf{I}
$$

and

$\mathbf{x}((k+1) n)=\mathbf{A}^{n} \mathbf{x}(k n)+y_{s} \quad(\forall k \geq 0)$,

it follows that

$$
\mathbf{x}(k n)=\mathbf{x}(0)+k y_{s} \quad(\forall k \geq 0) .
$$

If $y_{s} \neq 0$, then $\mathbf{x}(k n)$ will get unbounded for sufficiently large $k$. This contradicts the boundedness of $\mathbf{x}(k n)$. Thus, $y_{s}=0$.

1b) Let the trajectory stating with $\mathrm{x}(0)$ have the periodic symbolic sequence $\mathbf{s}_{u}$. Hence, this trajectory is determined by $\mathbf{x}(k+(m+1) L)=\mathbf{A}^{L} \mathbf{x}(k+m L)+\mathbf{\Psi}_{L} \mathbf{s}_{u}^{k} \quad(k<L)$.

Subtracting $\mathbf{z}_{k}$ from both sides of (25) yields

$$
\begin{aligned}
\mathbf{x} & (k+(m+1) L)-\mathbf{z}_{k} \\
& =\mathbf{A}^{L} \mathbf{x}(k+m L)+\mathbf{\Psi}_{L} \mathbf{s}_{u}^{k}-\mathbf{z}_{k} \\
& =\mathbf{A}^{L} \mathbf{x}(k+m L)+\left(\mathbf{I}-\mathbf{A}^{L}\right) \mathbf{z}_{k}-\mathbf{z}_{k} \\
& =\mathbf{A}^{L} \mathbf{x}(k+m L)-\mathbf{A}^{L} \mathbf{z}_{k} .
\end{aligned}
$$

When $L$ is not a multiple of $p, \mathbf{I}-\mathbf{A}^{L}$ is invertible. Thus, $\mathbf{z}_{i}$ is the unique solution of the set of equations (18). Following the Proof of Theorem 2, we have

$$
\mathbf{x}(k+m L)-\mathbf{z}_{k}=\mathbf{A}^{m L}\left(\mathbf{x}(k)-\mathbf{z}_{k}\right) .
$$

If $\mathbf{x}(0) \neq \mathbf{z}_{0}$, keep $k$ constant and let $m$ approach infinity. When $m L$ is a multiple of $n, \mathbf{A}^{m L}=\mathbf{I}$. Thus, the trajectory iterates itself. This equation implies a periodic trajectory with $n / L$ points on an ellipse centered at $\mathbf{z}_{k}$. For $k \in[0, L-1]$, there will be $L$ such elliptical sets. Since the maximum singular value of $\mathbf{A}$ is $(1+\cos (\Omega))^{1 / 2}$, then the length of the semimajor axis of these ellipses is

$(1+\cos (\Omega))^{\frac{1}{2}}\left\|\mathbf{x}(0)-\mathbf{z}_{0}\right\|=(1+\cos (\Omega))^{\frac{1}{2}} \rho$.

In addition, the semiminor axis is of length

$(1-\cos (\Omega))^{\frac{1}{2}}\left\|\mathbf{x}(0)-\mathbf{z}_{0}\right\|=(1-\cos (\Omega))^{\frac{1}{2}} \rho$.

If $\mathbf{x}(0)=\mathbf{z}_{0}$, then the trajectory of the system is

$$
\left\{\mathbf{z}_{0}, \mathbf{z}_{1}, \mathbf{z}_{2}, \ldots, \mathbf{z}_{L-1}, \mathbf{z}_{0}, \ldots\right\}
$$

which is period- $L$.

1c) If $L$ is a multiple of $p$, then $L=n=\operatorname{lcm}(L, p)$. Thus, following the result in 1a), we know that $L$ is the minimum period of the trajectory.

2) When $r$ is irrational. For a certain $k$, the trajectory determined by (27) is a dense elliptical set. Thus, for $k \in[0, L-1]$, there will be $L$ such sets.

Remark 3: In [8], the difficulty of finding patterns from the phase plane portrait was stated, particularly when $r$ is rational

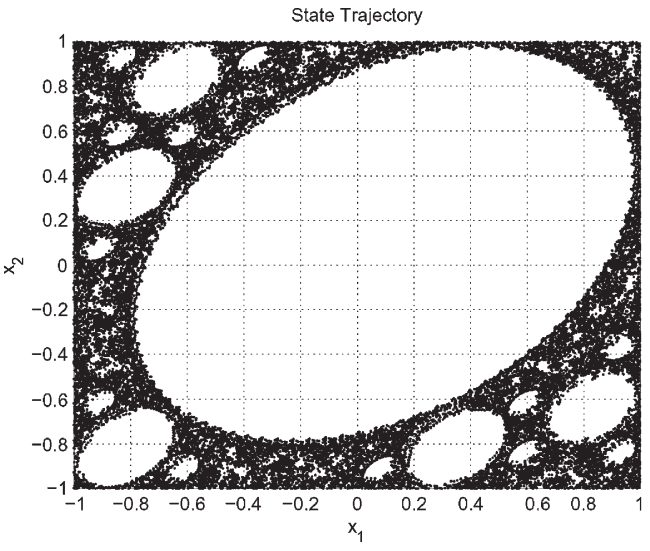

Fig. 3. Chaos-like state trajectory with initial state $\mathbf{x}(0)=$ $\left[\begin{array}{ll}-0.1458 & 0.9028\end{array}\right]^{T}, \omega=2 \pi / 3, \quad$ and $\Omega=1.2$. Calculation iterates 15000 times.

and the period of the symbolic sequence or the period of the input signal is large. Our framework provides a method to understand the periodic behaviors for the general periodic input case.

Remark 4: Theorem 3 reveals that the system trajectory is closely connected to the symbolic sequence and the value of $\Omega$. Periodic symbolic sequences imply ordered system dynamics, either discrete points scattered on the phase portrait when $\Omega$ is rational or finite dense elliptical sets when $\Omega$ is irrational. However, when the symbolic sequence is aperiodic, the system trajectory may exhibit irregular patterns, as shown in Fig. 3, which needs to be further studied.

\section{CONCLUSION}

In this brief, a framework for studying the dynamic behaviors of the second-order digital filter with two's complement arithmetic under general periodic inputs has been developed. Conditions for avoiding overflow have been derived. A mechanism for detecting periodic overflow dynamics has been established. Various periodic behaviors have been explored.

\section{REFERENCES}

[1] L. O. Chua and T. Lin, "Chaos in digital filters," IEEE Trans. Circuits Syst., vol. 35, no. 6, pp. 648-652, Jun. 1988.

[2] T. Lin and L. O. Chua, "On chaos of digital filters in the real world," IEEE Trans. Circuits Syst., vol. 38, no. 5, pp. 557-558, May 1991.

[3] Z. Galias and M. J. Ogorzalek, "On symbolic dynamics of a chaotic secondorder digital filter," Int. J. Circuit Theory Appl., vol. 20, no. 4, pp. 401-409, 1992.

[4] C. W. Wu and L. O. Chua, "Symbolic dynamics of piecewise-linear maps," IEEE Trans. Circuits Syst. II, Analog Digit. Signal Process., vol. 41, no. 6, pp. 420-424, Jun. 1994

[5] L. Kocarev, C. W. Wu, and L. O. Chua, "Complex behavior in digital filters with overflow nonlinearity: Analytical results," IEEE Trans. Circuits Syst. II, Analog Digit. Signal Process., vol. 43, no. 3, pp. 234-246, Mar. 1996.

[6] X. Yu and Z. Galias, "Periodic behaviors in a digital filter with two's complement arithmetic," IEEE Trans. Circuits Syst. I, Fundam. Theory Appl., vol. 48, no. 10, pp. 1177-1190, Oct. 2001.

[7] B. W. Ling, P. K.-S. Tam, and X. Yu, "Step response of a second-order digital filter with two's complement arithmetic," IEEE Trans. Circuits Syst. I, Fundam. Theory Appl., vol. 50, no. 4, pp. 510-522, Apr. 2003.

[8] B. W. Ling and P. K.-S. Tam, "Sinusoidal response of a second-order digital filter with two's complement arithmetic," IEEE Trans. Circuits Syst. I, Fundam. Theory Appl., vol. 50, no. 5, pp. 694-698, May 2003.

[9] B. W. Ling, Nonlinear Digital Filters: Analysis and Application. Oxford, U.K.: Academic, 2007. 